\title{
BIJECTIVE QUASI-ISOMETRIES OF AMENABLE GROUPS
}

\author{
TULLIA DYMARZ
}

\begin{abstract}
Whyte showed that any quasi-isometry between non-amenable groups is a bounded distance from a bijection. In contrast this paper shows that for amenable groups, inclusion of a proper subgroup of finite index is never a bounded distance from a bijection.
\end{abstract}

\section{INTRODUCTION}

In his book on infinite groups, Gromov $\mathrm{Gr}$, page 23] asked whether inclusion of finite index subgroups $F_{m} \longrightarrow F_{n}$ of two free groups is a bounded distance from a bi-Lipschitz map (i.e. a bijective quasi-isometry). Papasoglu answered this question affirmatively in $\mathrm{Pa}$. A more general question asked in [H, page 107] is whether any two groups which are quasi-isometric always have a bijective quasi-isometry between them? No counterexamples have been found.

Whyte showed in Wh that any quasi-isometry between non-amenable groups is a bounded distance from a bijection. In contrast, we will show that for amenable groups, inclusion of a finite index proper subgroup is never a bounded distance from a bijection (see Theorem 3.5). However, if the subgroup admits an " $n$-to- 1 " self quasi-isometry (where $n$ is the index of the subgroup) one can always compose this self map with the subgroup inclusion map to get a quasi-isometry that is a bounded distance from a bijective quasi-isometry. So for such groups commensurability implies that there does exist a bijective quasi-isometry.

\section{Preliminaries}

Definition 2.1. A map between metric spaces $f: X \longrightarrow Y$ is a quasiisometry if there exist $C, K \geq 0$ such that for all $x, y \in X$

$$
-C+\frac{1}{K} d(x, y) \leq d(f(x), f(y)) \leq K d(x, y)+C
$$

and there also exist $g: Y \longrightarrow X$ satisfying 2.1 such that $f \circ g$ and $g \circ f$ are a bounded distance from the identity. In this case $X$ and $Y$ are said to be quasi-isometric.

Date: June 10, 2018.

2000 Mathematics Subject Classification. 20F65.

Key words and phrases. infinite groups, geometric group thoery.

The author was supported in part by NSERC PGS B. 
Definition 2.2. A metric space $X$ is a uniformly discrete space of bounded geometry (UDBG space) if

- there is an $\epsilon>0$ such that, for all $x, y \in X, d(x, y)<\epsilon \Rightarrow x=y$ (i.e. $X$ is uniformly discrete), and

- for any $r>0$ there is a bound $M_{r}$ on the size of any $r$-ball in $X$ (i.e. $X$ has bounded geometry).

Example 2.3. A finitely-generated group with the word metric $d(x, y)=$ $\left\|x^{-1} y\right\|$ is a main example of a UDGB-space. The norm is defined with respect to a specific generating set so two different generating sets may give metric spaces which are not isometric but they are quasi-isometric.

Example 2.4. Any proper metric space admitting a properly discontinuous isometric cocompact group action is also a UDBG-space.

To define the notion of an amenable space we need some notation. Let $|S|$ denote the cardinality of the set $S$ and $\partial S=\{x \in X \quad 0<d(x, S) \leq 1\}$ denote the boundary of $S$.

Definition 2.5 (Folner Criterion). A UDBG-space is amenable if there exists a sequence of finite subsets $\left\{S_{i}\right\}$ with

$$
\lim _{i \rightarrow \infty} \frac{\left|\partial S_{i}\right|}{\left|S_{i}\right|}=0
$$

Such a sequence is called a Folner sequence.

We say a finitely generated group amenable if it is amenable as a UDBGspace with the word metric. Since amenability is preserved by quasi-isometries this is a well defined notion.

It will also be useful to review some conventions of big "O" notation.

Definition 2.6. We say

- $f(i)=O(g(i))$ if there exist $C, K>0$, such that $|f(i)| \leq C|g(i)|$ for all $i \geq K$.

- $O(f(i))<O(g(i))$ if $g(i) \neq O(f(i))$

- $O(g(i))=O(f(i))$ if both $g(i)=O(f(i))$ and $f(i)=O(g(i))$.

One important property we will use is that for $f, g \geq 0$

$$
O(f(i)+g(i))=O(f(i)) \text { if } g(i)=O(f(i)) .
$$

Using this notation, the statement of amenability can be rephrased as follows:

$X$ is amenable if there exists a sequence of finite sets $S_{i} \subset X$ such that $O\left(\left|\partial S_{i}\right|\right)<O\left(\left|S_{i}\right|\right)$. 


\section{UNIFORMLY FINITE HOMOLOGY}

The uniformly finite homology groups $H_{i}^{u f}(X)$ were first introduced by Block-Weinberger in BW1. Only $H_{0}^{u f}(X ; \mathbb{Z})$ is needed here, but for a detailed discussion of uniformly finite homology, see Wh or BW1.

For a UDBG-space $X$, let $C_{0}^{u f}(X)$ denote the vector space of infinite formal sums of the form

$$
c=\sum_{x \in X} a_{x} x
$$

where there exists $M_{c}>0$ such that $\left|a_{x}\right| \leq M_{c}$. Let $C_{1}^{u f}(X)$ denote the vector space of infinite formal sums of the form

$$
c=\sum_{x, y \in X} a_{(x, y)}(x, y)
$$

where there exist $M_{c}>0$ such that $\left|a_{(x, y)}\right|<M_{c}$ and $R_{c}>0$ such that $a_{(x, y)}=0$ if $d(x, y)>R_{c}$. Define a boundary map by

$$
\begin{aligned}
\partial: C_{1}^{u f}(X) & \longrightarrow C_{0}^{u f}(X) \\
(x, y) & \longmapsto y-x
\end{aligned}
$$

and extending by linearity. Then we let

$$
H_{0}^{u f}(X)=C_{0}^{u f}(X) / \partial\left(C_{1}^{u f}(X)\right)
$$

Some important facts about $H_{0}^{u f}(X)$ we will not prove here (see Wh] are

- if $X$ and $Y$ are quasi-isometric then $H_{0}^{u f}(X) \cong H_{0}^{u f}(Y)$.

- if $X$ is infinite then $H_{0}^{u f}(X)$ is a vector space over $\mathbb{R}$. (When $X$ is finite $\left.H_{0}^{u f}(X) \cong \mathbb{Z}\right)$

Definition 3.1. Any subset $S \subset X$ defines a class in $H_{0}^{u f}(X)$, denoted $[S]$, where $[\mathrm{S}]$ is the class of the chain $\sum_{x \in S} x$. We call $[X]$ the fundamental class of $X$ in $H_{0}^{u f}(X)$.

Using uniformly finite homology, Kevin Whyte developed in Wh a test to determine when a quasi-isometry between UDBG spaces is a bounded distance from a bijection.

Theorem 3.2 (Whyte). Wh Let $f: X \rightarrow Y$ be a quasi-isometry between $U D B G$-spaces. Then there exists a bijective map a bounded distance from $f$ if and only if $f_{*}([X])=[Y]$. (Here $\left.f_{*}([X])=\left[\sum_{x \in X} f(x)\right]\right)$

For non-amenable spaces we have the following theorem:

Theorem 3.3 (Block-Weinberger). BW1 Let $X$ be a UDBG-space. Then the following are equivalent:

- $X$ is non-amenable.

- $H_{0}^{u f}(X)=0$ 
- there exists $c=\sum_{x \in X} a_{x} x$ with $a_{x}>0$ such that $[c]=0$ in $H_{0}^{u f}(X)$

Some of the motivation behind Whyte's Theorem 3.2 was that combined with Theorem 3.3 it implies:

Any quasi-isometry between finitely generated non-amenable groups is a bounded distance from a bijection.

We can also use Theorem 3.2 to investigate quasi-isometries of amenable groups. To use Theorem 3.2 we need to be able to check when a chain in $c \in C_{0}^{u f}(X)$ represents the zero class in $H_{0}^{u f}(X)$. The following theorem gives such a criterion.

Theorem 3.4 (Block-Weinberger). BW1] (Theorem 7.6 in Wh) Let $X$ be a UDBG-space, and let $c=\sum_{x \in X} a_{x} x \in C_{0}^{u f}(X)$. Then we have $[c]=0 \in$ $H_{0}^{u f}(X)$ if and only if for any Folner sequence $\left\{S_{i}\right\}$,

$$
\left|\sum_{x \in S_{i}} a_{x}\right|=O\left(\left|\partial S_{i}\right|\right)
$$

We now show how Whyte's criterion can be used to show that subgroup inclusion for amenable groups is not a bounded distance from a bijection.

Theorem 3.5. Let $G$ be an amenable group with proper subgroup $H$ of finite index, i.e. $[G: H]=n>1$. Then the inclusion map $i: H \hookrightarrow G$ is not a bounded distance from a bijective map.

Proof. Using Theorem 3.4 we show that the chain $c=\sum_{x \in G \backslash H} x$ gives a nonzero class in $H_{0}^{u f}(G)$, that is $[c]=[G]-[H] \neq 0$. To this end let $\left\{S_{i}\right\}$ be any Folner sequence for $G$. Now $G=\bigcup_{k=1}^{n} g_{k} H$ and

so

$$
\sum_{k=1}^{n}\left|S_{i} \backslash g_{k} H\right|=(n-1)\left|S_{i}\right|
$$

$$
O\left(\left|S_{i}\right|\right)=O\left((n-1)\left|S_{i}\right|\right)=O\left(\sum_{k=1}^{n}\left|S_{i} \backslash g_{k} H\right|\right)=O\left(\left|S_{i} \backslash g_{k_{i}} H\right|\right)
$$

for some $k_{i}$. Let $F_{i}=g_{k_{i}}^{-1} S_{i}$. These sets also form a Folner sequence, since left multiplication by $g_{k_{i}}^{-1}$ is an isometry. Now $\left|g_{k_{i}}^{-1} S_{i} \backslash H\right|=\left|S_{i} \backslash g_{k_{i}} H\right|$. This gives us

$$
O\left(F_{i} \backslash H\right)=O\left(\left|F_{i}\right|\right)>O\left(\left|\partial F_{i}\right|\right) .
$$

So for the chain $c$,

$$
\left|\sum_{x \in F_{i}} a_{x}\right|=\left|F_{i} \backslash H\right|=O\left(\left|F_{i}\right|\right) \neq O\left(\left|\partial F_{i}\right|\right),
$$


and so $[c] \neq 0$.

The following is a shorter proof of Theorem 3.5 suggested by Weinberger.

Proof. For the inclusion map $i$ to be a bijection, Theorem 3.2 tells us that we need

$$
[H]=i_{*}([H])=[G] .
$$

But $[G]=n[H]$, so in fact we need $[H]=n[H]$. Now $G$ is amenable so $[H] \neq 0$. And since $H_{0}^{u f}(G)$ is torsion free $[H]=n[H]$ only if $n=1$.

Theorem 3.5 is actually a corollary of a more general result.

Theorem 3.6. If $\phi: H \longrightarrow G$ is a homomorphism of amenable groups with finite index image, $[G: \phi(H)]=n$, and finite kernel, $\left|\phi^{-1}(0)\right|=k$, then $\phi$ is a bounded distance from a bijection if and only if $n=k$.

Proof. As above, to get a bijection we need $\phi_{*}([H])=[G]$. But $\phi_{*}([H])=$ $k[\phi(H)]$ and $[G]=n[\phi(H)]$. Now $G$ is an amenable group, so $[\phi(G)] \neq 0$, giving us $n=k$.

\section{4. " $n$-TO-1" SELF QUASI-IsOMETRIES}

In this section we show how one can use subgroup inclusion to get bijective quasi-isometries between certain groups.

Definition 4.1. We call $f: X \longrightarrow X$ an " $n$-to-1" self quasi-isometry if $f$ is a quasi-isometry and $\left|f^{-1}(x)\right|=n$. In this case $f_{*}([X])=n[X]$.

Theorem 3.6] suggests that we may be able to "fix" subgroup inclusion $i: H \rightarrow G$ of index $n$ by precomposing with an $n$-to- 1 quasi-isometry $f$ : $G \rightarrow G$. Then $i \circ f: H \longrightarrow G$ is a new quasi-isometry that is a bounded distance from a bijection, since

$$
(i \circ f)_{*}([H])=i_{*}(n[H])=n[H]=[G] .
$$

This leads to the question:

Which amenable groups admit " $n$-to-1" quasi-isometries?

Example 4.2. It is easy to define an " $n$-to-1" quasi-isometry of $\mathbb{Z}$

$$
\begin{aligned}
\phi: \quad \mathbb{Z} & \rightarrow \mathbb{Z} \\
k & \mapsto\left\lfloor\frac{k}{n}\right\rfloor
\end{aligned}
$$

where $\left\lfloor\frac{k}{n}\right\rfloor$ denotes the greatest integer less than or equal to $\frac{k}{n}$. This is an " $n$-to- 1 " map of $\mathbb{Z}$ which is a $\left(\frac{1}{n}, 1\right)$ quasi-isometry. One can extend this idea to get an " $n$-to-1" map on $\mathbb{Z}^{m}$ by applying the above map to one of the coordinates.

$$
\phi\left(k_{1}, k_{2}, \cdots, k_{m}\right)=\left(\left\lfloor\frac{k_{1}}{n}\right\rfloor, k_{2}, \cdots, k_{m}\right)
$$


We now consider another class of examples.

Example 4.3. The solvable Baumslag Solitar groups are given by the presentation

$$
B S(1, m)=\left\langle a, b \mid a b a^{-1}=b^{m}\right\rangle .
$$

We can view $B S(1, m)$ as a union of cosets of the subgroup $\langle b\rangle \cong \mathbb{Z}$. By identifying each coset with $\mathbb{Z}$ we can define an "n-to-1" map in a similar way as we do for $\mathbb{Z}$

$$
f_{\alpha}: \alpha b^{i} \mapsto \alpha b^{\left\lfloor\frac{i}{n}\right\rfloor}
$$

where $\alpha$ is the coset representative. Picking a set $C$ of coset representatives gives us an "n-to-1" map of $B S(1, m)$

$$
f_{C}: g \mapsto b^{\left\lfloor\frac{i}{n}\right\rfloor} \alpha
$$

where $g=\alpha b^{i}$ for some $\alpha \in C$.

A priori, if we choose random coset representatives for each coset we may not get a quasi-isometry of $B S(1, m)$. It turns out that we can identify the cosets with $\mathbb{Z}$ in such a way so that the resulting map is actually a quasi-isometry.

In order to understand how to pick the coset representatives it is useful to review some ideas from [FM]. The group $B S(1, m)$ acts properly discontinuously and cocompactly on a metric 2-complex $X_{m}$, which is a warped product of a tree $T_{m}$ and $\mathbb{R}$. The tree $T_{m}$ is a $(m+1)$-valent directed tree with one "incoming edge" and $m$ "outgoing edges" at each node. There is a natural projection $X_{m} \rightarrow T_{m}$. The inverse image of a coherently oriented line, (a bi-infinite path in $T_{m}$ respecting the orientation) is a hyperbolic plane. Any time we refer to these embedded hyperbolic planes we will identify them with the upper half plane model of $H^{2}$. The inverse image of a vertex is a horocycle, (called a branching horocycle). We can pick the basepoint of $X_{m}$ to lie on a branching horocycle.

$X_{m}$ is actually the universal cover of a complex $C_{m}$ (see FM for details) whose fundamental group is $B S(1, m)$. The fundamental domain can be thought of as a "horobrick" $h_{m} \subset H^{2}$ defined by the region bounded by $0 \leq x \leq n$ and $1 \leq y \leq m$ so that the top of the horobrick has length 1 and the bottom has length $m$ (see figure 1).

We can define a quasi-isometry $i: B S(1, m) \rightarrow X_{m}$ by mapping e, the identiy of $B S(1, m)$, to the basepoint of $X_{m}$ and extending equivariantly. Then we can view $B S(1, m)$ as embedded in $X_{m}$ where group elements lie on branching horocycles and each branching horocycle contains a coset of $\langle b\rangle$. Elements which differ only by the generator $a$ are distance $\log (m)$ apart and lie on adjacent branching horocycles. Since $i$ is a quasi-isometry it has a coarse inverse. Let $j: X_{m} \rightarrow B S(1, m)$ be the coarse inverse of $i$ which maps each fundamental domain in $X_{m}$ to the unique element of $B S(1, m)$ in that domain. (i.e. each horobrick is mapped to the element in the upper left corner.) Any map $f: B S(1, m) \rightarrow B S(1, m)$ gives us a map 


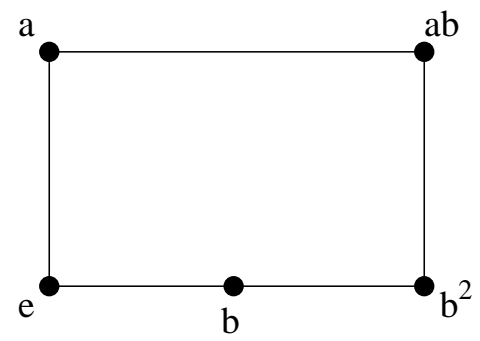

FiguRE 1 . the horobrick $h_{2}$ which is the fundamental domain corresponding to the element $a$ in $B S(1,2)$

$i \circ f \circ j: X_{m} \rightarrow X_{m}$ which is a quasi-isometry of $X_{m}$ if and only if $f$ is a quasi-isometry of $B S(1, m)$. When convenient we will make no distinctions between $f$ and $i \circ f \circ j$.

The key idea is to "line up" all of the cosets so that our map $f$, when restricted to each hyperbolic plane in $X_{m}$, is a bounded distance from the quasi-isometry

$$
\phi:(x, y) \longmapsto\left(\frac{1}{n} x, y\right) .
$$

To this end we need to consider another projection $X_{m} \rightarrow H^{2}$. From [FM] we know that there exists a unique map $\rho_{m}: X_{m} \rightarrow H^{2}$ with the following properties:

- $\rho_{m}$ takes horocycles to horocycles

- $\rho_{m}$ is an isometry when restricted to each hyperbolic plane in $X_{m}$

- $\rho_{m}$ is normalized to take the base point of $X_{m}$ to the point $(x, y)=$ $(0,1)$

Let $l=\left\{(0, y) \in H^{2}\right\}$ be the $y$-axis and consider $T=\rho^{-1}(l)$. $T$ intersects each branching horocycle at exactly one point. This will be our reference point which we will call "0". Since each branching horocycle "contains" a coset of $\langle b\rangle$, pick the coset representative for this coset to be a group element $\alpha$ which lies closest to "0". (There may be two such elements in some cases). Note that for each $\alpha$ the distance between $\alpha$ and $T$ is at most one. Our map $i \circ f_{\alpha} \circ j$ is bounded distance from the map $\phi$ when restricted to each branching horocycle. Because all of the $\alpha$ 's are a uniformly bounded distance from $T$ we have that for each hyperbolic plane $Q$ in $X_{m}$ the total map $f_{C}$ restricted to $Q$ is a bounded distance from $\phi$. So by the rubberband principle $i \circ f_{C} \circ j$ is a quasi-isometry of $X_{m}$ and so $f_{C}$ is an "n-to-1" quasiisometry of $B S(1, m)$.

We now consider one criterion for when a group does admit an " $n$-to-1" quasi-isometry. If $G$ contains a subset $G^{\prime}$ such that

$$
G=\bigsqcup_{i=1}^{n} G^{\prime} g_{i} \text {. }
$$


and there exists a bijective quasi-isometry

$$
f: G^{\prime} \longrightarrow G
$$

then $f$ extends to an " $n$-to- 1 " self quasi-isometry of $G$ given by

$$
\begin{aligned}
f^{\prime}: G & \longrightarrow G \\
g^{\prime} g_{i} & \longmapsto f\left(g^{\prime}\right) \quad\left(g^{\prime} \in G^{\prime}\right)
\end{aligned}
$$

This holds in particular if $G^{\prime}$ is a subgroup of $G$ with $\left|G: G^{\prime}\right|=n$ and $f: G^{\prime} \rightarrow G$ is an isomorphism.

If it were possible to find an " $n$-to- 1 " self quasi-isometry for all amenable groups, then we would have a bijective quasi-isometry between any two commensurable, amenable groups.

\section{Acknowledgments}

I'd like to thank Kevin Whyte for useful conversations, Shmuel Weinberger, and especially my advisor Benson Farb for many helpful suggestions.

\section{REFERENCES}

[BS] Bieri, R, Strebel, R., Almost finitely presented soluble groups Comment. Math. Helv., 53 (1978), p. 258-278

[BW1] Block, J.,Weinberger, S., Aperiodic tilings, positive scalar curvature and amenability of spaces J. Amer. Math. Soc. 5 (1992), no. 4, p. 907-918

[BW2] Block, J., Weinberger, S., Large scale homology theories and geometry AMPS/IP Studies in Advanced Mathematics, 2 1997. p. 522-569

[FM] Farb, B., Mosher, L., A rigidity theorem for the solvable Baumslag-Solitar groups, Inventiones mathematicae, Springer-Verlag 1998. p. 419-451

[Gr] Gromov, M. Asymptotic invariants of infinite groups, in Geometric group theory, Vol. 2 (Sussex, 1991), 1-295, Cambridge Univ. Press, Cambridge, 1993

[H] P. de la Harpe, Topics in Geometric Group Theory Chicago, 2000

[Pa] Papasoglu, P., Homogeneous trees are bi-Lipschitz equivalent Geom. Dedicata 54 (1995), no. 3, p. 301-306

[S] Schupp, Paul E., Some reflections on HNN extensions Proceedings of the Second International Conference on the Theory of Groups (Australian Nat. Univ., Canberra, 1973), p. 611-632. Lecture Notes in Math., Vol. 372, Springer, Berlin, 1974.

[Wh] Whyte, K., Amenability, Bilipschitz Equivalence, and the Von Neumann Conjecture Duke Journal of Mathematics 1999, p. 93-112

Department of Mathematics, University of Chicago, Chicago, Illinois 60637

E-mail address: tullia@math.uchicago.edu 